\title{
Framework for Multimodal Interfaces in Conversation of Man with Man-Made Machine
}

\author{
${ }^{1}$ Dr. S. Sai Satyanarayana Reddy, ${ }^{2}$ Dr. Ramesh Shahabadkar \\ ${ }^{1,2}$ Department of Computer Science and Engineering, Vardhaman College of Engineering, Hyderabad \\ Email: ramesh.shahabadkar@gmail.com
}

\section{Received: 09 ${ }^{\text {th }}$ July 2018, Accepted: $14^{\text {th }}$ August 2018, Published: $31^{\text {st }}$ August 2018}

\begin{abstract}
The main motto of writing this paper is to give a detailed description about the content of how a man creates and interacts with the machine or system designed by the creative and technical ways with the intelligence of man. This detailed description consists of the knowledge of what the primary requirements are, regarding the technology available and being used and the advancement that is taking place in the various areas of work such as the architectural field, to design a system. Usually the architecture of man and man-made communication system is of two types. They are the unimodal and the multimodal.
\end{abstract}

Keywords: Man, Man-made Communication, Unimodal, Multimodal

\section{Introduction}

Appropriate use of the machines had often consolidated the questioning from man. The manner of communicating that is caused by the man with the machine has run over a deep pathway. The adventure is quiet and is being processed with the construction of brand-new patterns for the technological machines that are coming along in a large number in the coming up generations which is on a fast run. The advancement in the field of communication between the man and manmade machines is not only regarding the communication, but also gives contact with various other fields including the history. Rather than the designing of formal models, a different pattern of works based on the researches being done on multivariate systematic work comparative for the invariant systematic work. And, the smart modification is being preferred compared to the ordered modification.

\section{Man and Man-Made Communication Wording}

Consistently termed as the Human-Computer communication. Obeying the interfacing rules undoubtedly by materializing the upcoming development of the machines which can be fit for use for man significantly. The elementary dispute represents the important conditions which are to be taken into application for the construction of the performance and usage. The reason for the construction of system can describe the use and need i.e. what the system does for the accomplishment of its purpose. The performance of the system describes the group of steps that are to be provided to the customers. The usage of the system is defined based upon the performance i.e. depending upon the dimension and extent of its efficiency for the accomplishment of their objectives. The construction of a good man-made machine is based on the subjective and context. The major steps include the statements, virtual reality, graphical user interface which act as the different tools for the software.

\section{Related Work}

The advances made in the last decade in HCI have almost made it impossible to realize which concept is fiction and which is and can be real. The thrust in research and the constant twists in marketing cause the new technology to become available to everyone in no time. However, not all existing technologies are accessible and/or affordable by the public. In the beginning portion of this section, a description of the technology that is available to and used by public is confronted. In the second section, an outlook of the way to which HCI research is heading has been withdrawn.

\section{Existing Man-Made Technologies}

Man-Made proposal must take numerous facades of human activities and requirements to be serviceable. The complication of the grade of the contribution of a human in communication with a vehicle is occasionally indiscernible associated to the acceptance of the interface technique. The prevailing interfaces vary in the grade of complication together since of grade of operability and the monetary and cost-effective feature of the engine in the marketplace. For example, an electrical pot need not to be cultured in the interface as its mere operability is to warmth the water and it might not be economical to require an interface additional than a thermo-static switch-on and switch-off. On the contrary, a modest web -site that might be limited in operability must be intricate adequate in use to entice and retain clienteles. 
Thus, in scheming of Man-Made technologies, the approach of action that indicates a client through an engine must be systematically measured. The operator action takings in three dissimilar approaches: physical, perceptive, and sentimental. The physical feature governs the mechanism of interface amid human and system although the perceptive feature transactions with customs that operators may comprehend the computer and interrelate through it. The sentimental feature is an extra current subject matter and it attempts not solitary to brand the communication a pleasing practice for the operator, nonetheless likewise to mark the operator in a technique that brand operator endure to usage the system by altering attitudes and sentiments in the direction of the operator.

The emphasis of the paper is typically on the improvements in physical feature of communication and to display in what way diverse approaches of collaboration may be shared (Multi-Modal Interaction) and in what way every technique may be better-quality in presentation (Intelligent Interaction) to deliver an improved and convenient interface for the operator. The prevailing physical know-hows for Man-Made techniques fundamentally may be characterized by the comparative social sense that the engine is planned for. The systems are fundamentally depend on three senses of human-being: visualization, touching, and aural.

Peripheral components that depend on visualization stand the utmost used kind and are normally which are pointing components. The pointing components are any kind of interface that practices keys and knobs alike a control panel. The pointing components specimens are mouse, touch screen panels, graphic tablets, joysticks, trackballs, and pen-based input. The output components can be any kind of VDU display or laser printing device. The components that depend on trial are extra improvement components that frequently essential particular kind of dialogue recognition. These components target to enable the communication as ample as imaginable and consequently, are ample hard to figure out. Output hearing components are though at ease to produce. These days, altogether kind of nonhearing and hearing pointers and communications are shaped by machineries as output pointers. Honks, alarms, and step-by-step map reading guidelines of a Global Positioning System components are modest samples.

The maximum problematic and expensive components to shape are haptic components. "These classes of interfaces produce senses to the human body through touch, mass and comparative inflexibility." Haptic components are normally made for simulated realism or frailty assistive claims.

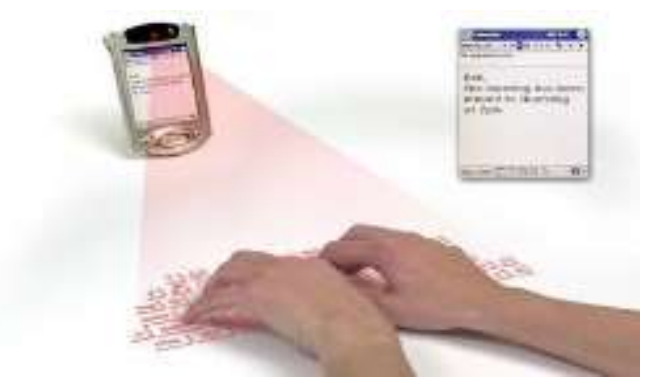

Figure 1: Canesta Simulated Control Panel

\section{Man-Made Systems Architecture}

Utmost significant feature of a Man -Made proposal is its formation. In datum, any assumed interface is normally well -defined by the quantity and assortment of inputs and outputs the aforementioned offers. Architecture of a Man -Made scheme demonstrates whatever the inputs and outputs are and in what way they are composed.

\section{Unimodal Man-Made Systems}

An interface largely depend on amount and assortment of its inputs and outputs which are communiqué conduits that permit operators to interrelate through workstation via interface. Every self -governing solitary conduits is called a modality. A scheme that is grounded on solitary single modality is called unimodal. Built on the nature of dissimilar modalities, they can be divided into three categories:

1. Graphical-Based

2. Auditory-Based

3. Sensor-Based

\section{Graphical-Based}

The graphical based user computer communication is possibly the maximum extensive part in Man-Made investigation. Considering the degree of claims and diversity of uncluttered glitches and tactics, investigators strained to challenge dissimilar features of operator replies which may be familiar as a graphical pointer.

\section{Facemask Appearance Study Physique Measure Pursuing Motion Acknowledgment Look Discovery \\ Auditory-Based}

The auditor-based communication amid a computer and an operator is extra zone of Man-Made systems. This zone pacts through statistics assimilated by diverse auditory pointers. Although the behavior of auditory pointers cannot be as mutable as graphical pointers, 
nonetheless the statistics assembled since auditory pointers may be additional trustable, obliging, and is particular instances exclusive offerers of statistics.

$$
\begin{aligned}
& \square \quad \text { Dialogue Recognition } \\
& \square \quad \text { Orator Recognition } \\
& \square \quad \text { Aural Sentiment Study } \\
& \square \quad \text { Man-Made Sound }
\end{aligned}
$$

Sensor-Based

This segment is a grouping of diversity of zones through an extensive variety of claims. The cohesion of these dissimilar zones is that at least one physical sensor is used among operator and engine to offer the communication. These sensors as exposed below can be very primitive or very stylish.

\section{Applications}

A standard specimen of a multimodal scheme is the "Keep That Here" demonstration scheme. This scheme allowable unique to change an item into a novel position on a plot on the awning by maxim "Keep that Here" though pointing to the item itself before pointing to the anticipated terminus. Multimodal interfaces need to be used in an amount of claims with graph-based simulations, such as information kiosks and biometric confirmation schemes.

Multimodal interfaces may propose a quantity of compensations over outdated interfaces. For one thing, they may propose an extra usual and operator-friendly familiarity. For example, in a construction industry system called Real Hunter, one may point through a finger to a household of notice and express to kind enquiries around that specific household. By means of a pointing signal to choice an article and by means of speech to make enquiries around it exemplifies the category of normal involvement multimodal interfaces propose to their operators. Additional main strong point of multimodal interfaces is their capability to offer redundancy to lodge diverse folks and diverse situations. For example, Kiosk allows one to use speech or writing to postulate the sort of commercial to exploration for on a chart. Therefore, in a loud set, one can deliver input through script-writing somewhat than dialogue.

$\begin{array}{ll}\square & \text { Driver Monitoring } \\ \square & \text { Intelligent Homes/Offices } \\ \square & \text { Smart Video Conferencing } \\ \square & \text { E-Commerce } \\ \square & \text { Intelligent Games }\end{array}$
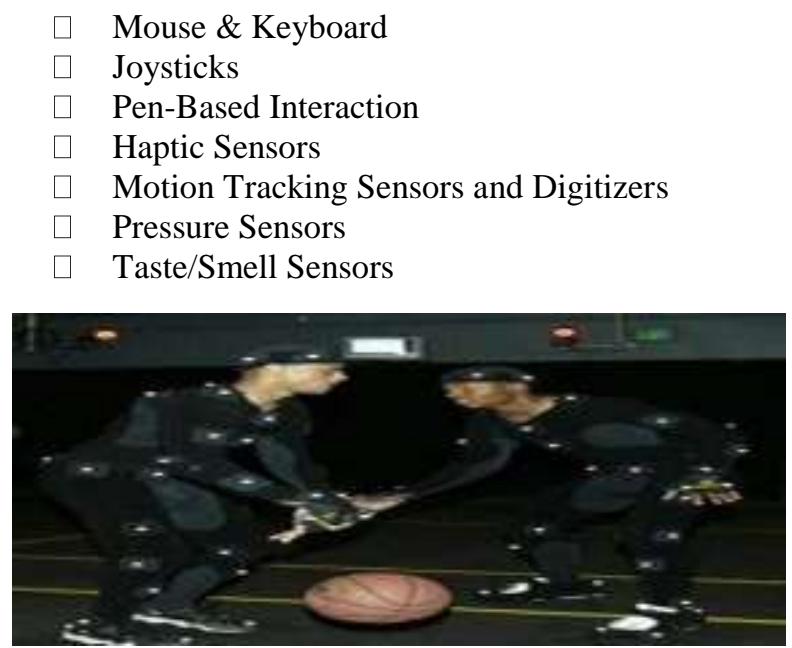

Figure 2: Wearable Gesture Seizure Fabric for Making of Audiovisual Competitions

$\square \quad$ Helping People with Disabilities

\section{Multimodal Systems for Differently Abled People} One virtuous claim of multimodal schemes is to discourse and contribution differently abled persons, which necessity additional classes of interfaces than normal persons. In such schemes, differently abled persons may act on task on the Personal Computer by interrelating through the engine by dialogue and skull actions.

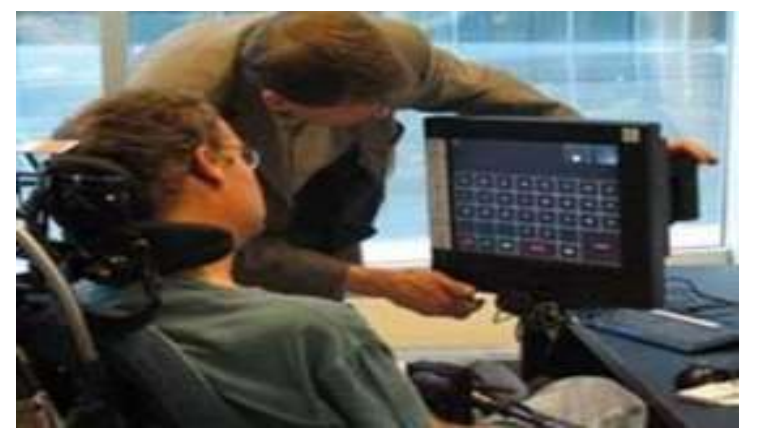

Figure 3: Look Discovery Pointing System for Differently Abled Persons

Dual modalities are used: dialogue and skull actions. Together modalities are vigorous uninterruptedly. The skull position designates organizes of the pointer in present period instant on the monitor. Dialogue delivers the desirable evidence around the connotation of the deed that should be achieved with an article designated by the pointer.

Synchronization amid the dual modalities is achieved by scheming the pointer position at the commencement of dialogue discovery. This is mostly 
outstanding to the statistic that throughout the procedure of articulating the comprehensive text sentence, the pointer position may be stimulated by poignant the skull, and before the pointer may be pointing to additional visual entity; furthermore, the knack which should be content is looked in the mind of a user in a small period beforehand start of idiom response

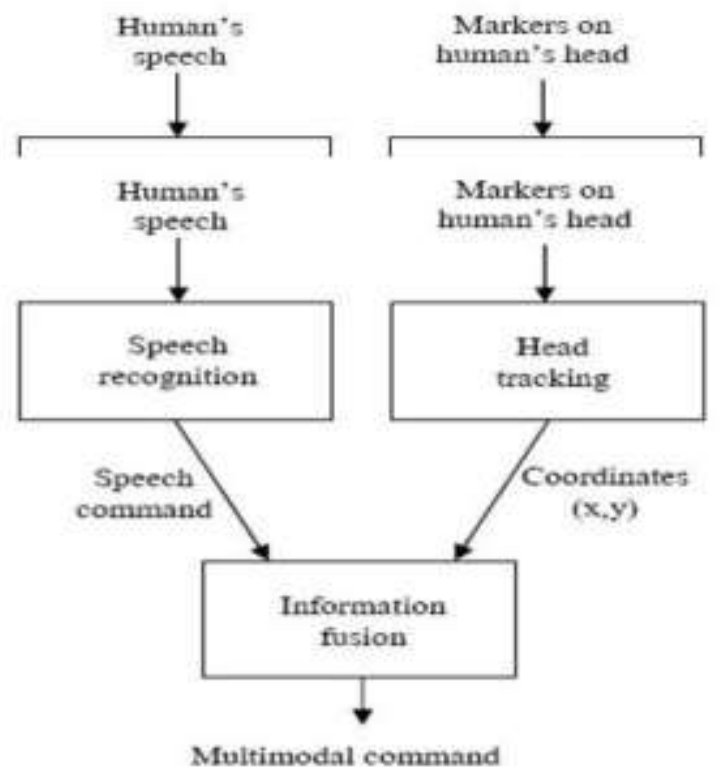

Figure 5: Diagram of a Bimodal System Sentiment Acknowledgment Multimodal Systems A world full of computers are more and more omnipresent, it is vital that engines recognize and understand all implicit hints and explicit hints, that we might deliver them concerning our purposes. A normal man-machine communication cannot be grounded exclusively on obviously specified instructions. Machines drive to distinguish the numerous interactive indications grounded on which to conjecture one's sensitive state. This is a noteworthy portion of the enigma that one should place organized to forecast precisely one's purposes and upcoming comportment. Users may type forecast around one's sensitive state built on their annotations around one's face, human body, and speech. Though, the accurateness may be better-quality by $36 \%$ after user juries are assumed entree to together façade and human-body modalities organized [66]. This proposes that distress acknowledgment, which takes intended maximum portion absorbed on facemask expressions, may significantly profit after multimodal synthesis practices. The recorded four sentiments: "grief, annoyance, pleasure, and impartial state". The thorough facemask gestures remained apprehended in combinations through concurrent dialogue footages. Abstracted trials displayed that the routine of the facemask recognition-based method overwhelmed the one grounded on aural evidence singular. Consequences likewise demonstration that a suitable synthesis of together modalities provided quantifiable developments. Consequences demonstration that the sentiment acknowledgement method grounded on aural evidence solitary spring a general presentation of 71 percent, associated to a complete action of 86 percent for an acknowledgment method based on facemask terms. This is owing to the datum that the impudence zones spring significant evidence for sentiment cataloguing.

\section{Multimodal Man-Robot Interface Applications}

The man and robot interfaces regularly want to carry mechanisms to pointing to precise sights and for enunciating procedure inventing requirements. The preceding grouping of message is well housed by gestures, although the latter is improved considerate by negotiation. Therefore, the man-robot interface factory-made by the Naval Research Laboratory (NRL) should originate as no astonishment. NRL's interface permits persons to argument to a position although proverb "Go over there". Furthermore, it permits operators to usage a PDA display as a third conceivable opportunity of communication, which might be resorted to after dialog or finger gesticulation acknowledgment is deteriorating. Additional multimodal man-robot interface is the single erected by Interactive System Laboratories (ISL), which permits use of dialogue to appeal the robot to do somewhat though gesticulations might be used to point to substances that are mentioned to by the dialogue. Moreover, in ISL's interface, the scheme may request for explanation from the operator once hesitant around the input.

\section{Conclusion}

The communication of man with the machine designed by man, i.e., the man-made machine is very essential segment in the arrangement of systems. The characteristics of the designed system are stated based upon the way it is shown and its functional operation during the time of work. Thus, much consideration is needed to demonstrate an improvement in the pattern of projecting. A unique way to exhilarate is to read over the ordinary approach for cooperation among the smart, flexible and the logical ways. Smart way to sort 
Helix Vol. 8(5): 3716- 3720

and see out a flood to enclose the modern technological ways into the surroundings to create more general and imperceptible ways at the same minute. For the communication of man with the machine is being developed from day by day around virtual reality. The present paper is to bring home the bacon with all the detailed description regarding the main content and the analysis through broad reference ballot.

\section{References}

[1] D. Te'eni, J. Carey and P. Zhang, Human Computer Interaction: Developing Effective Organizational Information Systems, John Wiley \& Sons, Hoboken (2007).
[2] B. Shneiderman and C. Plaisant, Designing the User Interface: Strategies for Effective HumanComputer Interaction (4th edition), Pearson/AddisonWesley, Boston (2004).

[3] J. Nielsen, Usability Engineering, Morgan Kaufman, San Francisco (1994).

[4] A. Chapanis, Man Machine Engineering, Wadsworth, Belmont (1965).

[5] R.W. Picard, Affective Computing, MIT Press, Cambridge (1997).

[6]J.S. Greenstein, "Pointing devices", in M.G. Helander, T.K. Landauer and P. Prabhu (eds), Handbook of Human-Computer Interaction, Elsevier Science, Amsterdam (1997) 